\section{Tumores hepáticos primarios malignos no hepatocarcinoma ni colangiocarcinoma}

\author{
GONZALO ARANEDA ${ }^{1}$, RAFAEL PONIACHIK ${ }^{2, \mathrm{a}}$, \\ ALEJANDRO FREUNDLICH ${ }^{2, a}$, LAURA CARREÑO ${ }^{3}$, JAIME PONIACHIK $^{1,4}$
}

\section{Primary malignant hepatic tumors other than hepatocarcinoma or cholangiocarcinoma. A series of cases}

Background: There are several types of primary malignant hepatic tumors (PMHT) other than hepatocellular carcinoma (HCC) and cholangiocarcinoma (CC): they are infrequent and poorly known. Imaging studies could help characterize the lesions and may guide the diagnosis. However, the definitive diagnosis of PMHT is made by pathology. Aim: To review a registry of liver biopsies performed to diagnose hepatic tumors. Patients and Methods: Review of a pathology registry of liver biopsies performed for the diagnosis of liver tumors. Among these, 25 patients aged $57 \pm 17$ years, $60 \%$ males, in whom a liver tumor other than a HCC or CC was diagnosed, were selected for review. The medical records of these patients were reviewed to register their clinical characteristics, imaging and the pathological diagnosis performed during surgery and/ or with the percutaneous liver biopsy. Results: Ten patients (40\%) had neuroendocrine tumors, six (24\%) had a lymphoma and four (16\%) had hepatic hemangioendothelioma. Angiosarcoma and sarcomatoid carcinoma were diagnosed in one patient each. In 22 patients (88\%), neither clinical features nor imaging studies gave the correct diagnosis. Four patients (16\%) had chronic liver disease. The most frequent symptoms were weight loss in $28 \%$ and abdominal pain in 24\%. Conclusions: The most common PMHT other than HCC and CC were neuroendocrine tumors and lymphomas. Imaging or clinical features were not helpful to reach the correct diagnosis.

(Rev Med Chile 2019; 147: 751-754)

Key words: Liver Neoplasms; Magnetic Resonance Imaging; Neoplasms, Unknown Primary; Tomography, X-Ray Computed.

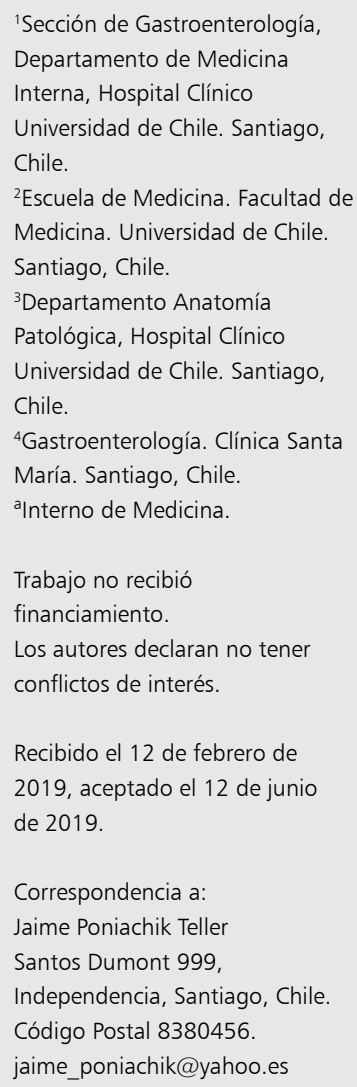

Correspondencia a: Jaime Poniachik Teller Santos Dumont 999, Independencia, Santiago, Chile. Código Postal 8380456 jaime_poniachik@yahoo.es

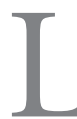

os tumores hepáticos malignos hepatocarcinoma (HCC) y colangiocarcinoma $J$ (CC) representan alrededor de 95\% de los cánceres hepáticos primarios. Sin embargo, existen múltiples otros tumores poco conocidos y subdiagnosticados ${ }^{1-3}$.

Las neoplasias hepáticas malignas primarias (NHMP) no HCC no CC derivan del tejido mesenquimático como hemangioendotelioma epitelioide, linfoma primario, tumores neuroen- docrinos, carcinoma sarcomatoide, angiosarcoma y sarcoma ${ }^{1-5}$. El diagnóstico de NHMP no HCC no CC es complejo, podría sospecharse por imágenes, sin embargo, la biopsia hepática y el estudio inmunohistoquímico entregan el diagnóstico definitivo en la mayoría de los $\operatorname{casos}^{1-4}$.

Actualmente existen escasos reportes sobre NHMP no HCC no CC, por lo que consideramos importante mostrar la casuística de 17 años de un centro universitario en Santiago, Chile. 


\section{Pacientes y Métodos}

Se realizó un estudio retrospectivo descriptivo, se consideraron todas las biopsias hepáticas realizadas entre 1999 y 2016, disponibles por tumor hepático en los archivos del Departamento de Anatomía Patológica del Hospital Clínico Universidad de Chile. Se revisaron informes histopatológicos de biopsias hepáticas obtenidas quirúrgicamente o por punción percutánea. Se ingresaron al estudio solo aquellas biopsias con diagnóstico histopatológico de NHMP no HCC ni $\mathrm{CC}$, correspondientes a 28 pacientes, obteniéndose información completa en 25 de ellos.

Se revisaron historias clínicas e informes histopatológicos de los pacientes seleccionados, se recolectaron variables clínicas y demográficos como sexo, edad, antecedentes mórbidos, sintomatología, diagnósticos imagenológicos y diagnóstico definitivo por estudio histopatológico. Se utilizó estadística descriptiva para mostrar resultados. El trabajo fue aprobado por Comité de Ética del Hospital Clínico Universidad de Chile.

\section{Resultados}

Se registraron 28 pacientes con NHMP no HCC no CC, 25 de ellos con datos completos, $60 \%$ sexo masculino, edad $57 \pm 17$ años, las principales comorbilidades fueron hipertensión arterial y diabetes mellitus tipo 2 (Tabla 1). Los subtipos más frecuentes (Tabla 2) fueron $40 \%$ tumores neuroendocrinos (10/25) y $24 \%$ linfoma $(6 / 25)$. En $88 \%$ de los casos, el diagnóstico no fue sospechado por antecedentes clínicos ni estudios imagenológicos.

Solo tres tumores fueron diagnosticados correctamente previo a la biopsia, basados en antecedentes clínicos y hallazgos imagenológicos: un hemangioendotelioma epiteloideo, un linfoma primario y un angiosarcoma. En nuestra serie, 4/25 (16\%) casos presentaron enfermedad hepática crónica detectados por imágenes abdominales de control. Los síntomas más frecuentes fueron: $28 \%$ disminución de peso (7/25) y $24 \%$ dolor abdominal (6/25); en menor proporción, se registró ictericia, náuseas, distensión abdominal, vómitos y diarrea.

Los tumores neuroendocrinos primarios hepáticos fueron las NHPM no HCC no CC más frecuentes de nuestra serie, con una proporción hombre: mujer de 1:1, con promedio de edad 65 años (rango: 48-86 años). Todos nuestros casos fueron no funcionantes, siendo la manifestación más frecuente ( $50 \%$ de los casos) presencia de una masa abdominal. Solo en dos se lograron identificar lesiones hipervasculares que serían características, en ambos casos con lesiones múltiples, el estudio histológico se consideró como definitivo.

El linfoma primario del hígado fue la segunda NHPM no HCC no CC más frecuente de nuestra serie. En esta casuística, un paciente tenía infección por virus de la inmunodeficiencia humana (VIH), uno sarcoma de Kaposi, uno había recibido trasplante hepático y renal y otro tenía antecedente de leucemia linfática crónica, y solo dos no presentaron antecedentes. Todos nuestros casos fueron hombres, con una edad promedio 65 años (rango: 47-82 años). Para el diagnóstico definitivo, en todos los casos fue necesaria la biopsia, ya que fueron inespecíficas las pruebas de laboratorio e imágenes.

\section{Tabla 1. Antecedentes mórbidos descritos} con los NHPM no HCC ni CC

\begin{tabular}{|lc|}
\hline Antecedentes mórbidos & n de casos \\
\hline Hipertensión arterial & 10 \\
\hline Diabetes mellitus 2 & 5 \\
\hline Tabaquismo & 4 \\
\hline Insulinoresistencia & 2 \\
\hline VIH & 2 \\
\hline Pancreatitis & 2 \\
\hline Hipotiroidismo & 2 \\
\hline Cirrosis & 3 \\
\hline
\end{tabular}

Tabla 2. Distribución de subtipos de NHPM no HCC no CC

\begin{tabular}{|l|c|}
\hline Tipos de tumores & $\begin{array}{c}\text { n de casos } \\
(\mathbf{n}=\mathbf{2 5})\end{array}$ \\
\hline Neuroendocrino & $40 \%(10)$ \\
\hline Linfoma & $24 \% \quad(6)$ \\
\hline Hemangioendotelioma & $16 \% \quad(4)$ \\
\hline Sarcoma & $12 \% \quad(3)$ \\
\hline Angiosarcoma & $4 \% \quad(1)$ \\
\hline Carcinoma sarcomatoide & $4 \% \quad(1)$ \\
\hline
\end{tabular}


El tercer tumor más frecuente fue el hemangioendotelioma epiteloideo. En los cuatro casos la proporción hombre: mujer fue 1:1, con edad promedio de 41 años (rango: 27-50 años). El 75\% (3/4) de los casos fueron asintomáticos, siendo el diagnóstico definitivo con biopsia. Cuatro pacientes han sido trasplantados. En nuestra serie se encontró una incidencia de daño hepático crónico, determinado por imágenes, en 14\% de los casos.

Los otros subtipos de NHPM no HCC no CC fueron significativamente menos frecuentes, tres casos de sarcomas, uno de angiosarcoma y uno de carcinoma sarcomatoide, representan 20\% de los casos. En todos los pacientes el diagnóstico se pudo definir solo con estudio histológico, dada la baja especificidad de la clínica y los hallazgos imagenológicos.

\section{Discusión}

Las NHPM distintos a HCC y CC son infrecuentes y de difícil diagnóstico. En nuestra casuística fueron identificados 28 casos de NHMP no HCC no CC en los registros de 16 años en un centro universitario de referencia nacional y fueron incluidos en este reporte solo los 25 pacientes con datos completos. Las NHMP no HCC no CC más frecuentes fueron los tumores neuroendocrinos primarios, con $40 \%$ de los casos, seguidos de los linfomas (24\%) y hemangioendotelioma (16\%), frecuencias similares a otros reportes ${ }^{1-6}$.

En esta serie, solo $12 \%$ de los casos hubo una sospecha clínica y diagnóstico correcto previo a la biopsia hepática (hemangioendotelioma, linfoma, angiosarcoma), determinándose fundamentalmente por los hallazgos en estudios de imágenes abdominales dinámicas (tomografía axial computada, resonancia magnética o ambos). El diagnóstico definitivo de este tipo de lesiones es por estudio histológico con técnicas de inmunohistoquímica de forma asociada.

Los tumores neuroendocrinos primarios hepáticos fueron las NHPM no HCC no CC más frecuentes de nuestra serie. De los diez pacientes con confirmación histológica de tumor neuroendocrino hepático, observamos una relación 1:1 según sexo, edad entre 48 y 86 años, lo cual es concordante con lo reportado ${ }^{2-6}$, aunque en algunas series se demuestra una mayor frecuencia en mujeres. Todos nuestros casos fueron no funcionantes, siendo la manifestación más frecuente $(50 \%$ de los casos) presencia de una masa abdominal.

Además, en solo dos casos se lograron identificar lesiones hipervasculares que podrían ser características de este tipo de tumor, en ambos casos con lesiones múltiples ${ }^{1}$, sin embargo, también se encontraron imágenes atípicas como lesiones hipovasculares y en un caso se sospechó erróneamente neoplasia vesicular, siendo nuevamente el estudio histológico como definitivo.

El linfoma primario del hígado fue la segunda causa más frecuente de la serie con $24 \%$ de los casos. Según la literatura, este tipo de tumor se presenta más frecuentemente en contexto de inmunosupresión ${ }^{2-6}$. Para el diagnóstico definitivo en todos los casos fue necesaria la biopsia de la lesión, siendo inespecíficos clínica, laboratorio e imágenes, como ha sido reportado anteriormente $^{2-6}$.

En nuestra serie se determinó por imágenes el daño hepático crónico, observándose en 14\% de los casos. Este dato puede estar en relación a la amplia disponibilidad de estudios imagenológicos en los pacientes cirróticos; es relevante dado que señala que no todo tumor en hígado cirrótico corresponde a un HCC o CC, sobre todo cuando las imágenes no son características en mostrar los signos típicos de estos tumores. Lo anterior obliga al estudio diferencial dirigido, dado que algunos pacientes son subsidiarios de terapias quirúrgicas.

Los otros subtipos de NHPM no HCC no CC fueron mucho menos frecuentes, tres casos de sarcomas, uno de angiosarcoma y uno de carcinoma sarcomatoide, que representan $20 \%$ de nuestros casos, comparable a lo reportado en la literatura $^{7-10}$. En todos estos casos el diagnóstico se pudo definir solo con estudio histológico, dada la baja especificidad de la clínica y los hallazgos imagenológicos.

\section{Conclusión}

Las NHPM no HCC no CC son poco frecuentes y determinan un desafío diagnóstico por su presentación en la mayoría de los casos de forma asintomática y a la poca especificidad de los hallazgos imagenológicos. En esta serie, los tumores más frecuentes fueron los tumores neuroendocrinos, los linfomas y los hemangioendoteliomas epiteloideo, fundamentándose el diagnóstico en 
la histología de la lesión y su tratamiento de ser posible, es quirúrgico.

\section{Referencias}

1. Nikfarjam M, Muralidharan V, Christophi C. Primary hepatic carcinoid tumours. HPB (Oxford) 2004; 6: 137.

2. Balduzzi C, Yantorno M, Mosca Iván, Apraiz M, Velázquez $M$, Puente M, Moragrega $V$, et al. Linfoma hepático primario: causa infrecuente de lesión focal hepática. Acta Gastroenterol Latinoam 2010; 40 (4): 361-6.

3. Avlonitis VS, Linos D. Primary hepatic lymphoma: a review. Eur J Surg 1999; 165 (8): 725-9.

4. Steller EJ, van Leeuwen MS, van Hillegersberg R, Schipper ME, Rinkes IH, Molenaar IQ. Primary lymphoma of the liver - A com- plex diagnosis. World J Radiol 2012; 4 (2): 53-7.

5. Delshad SD, Ahdoot JJ, Portocarnero DJ. Primary hepa- tic lymphoma. Clin Gastroenterol Hepatol 2010; 8 (5): e49-e50.

6. Mouna B, Wafae A, Hind M, Hassan E. Primary liver lymphoma: a case report and literature review. J Cancer Ther 2011; 2 (5): 725-7.

7. Mehrabi A, Kashfi A, Fonouni H, Schemmer P, Schmied BM, Hallscheidt P, et al. Primary malignant hepatic epithelioid hemangioendothelioma. Cancer 2006; 107 (9): 2108-21.

8. Wang QB, Cui BK, Weng JM, Wu QL, Qiu JL, Lin XJ. Clinicopathological characteristics and outcome of primary sarcomatoid carcinoma and carcinosarcoma of the liver. J Gastrointest Surg 2012; 16 (9):1715-26.

9. Flores Rivera OI, Quintana Quintana M, Frias Aguirre YN, González Cervantes JG, Baena Ocampo L. Angiosarcoma hepático: reporte de un caso y revisión bibliográfica. Med Int Mex 2012; 28 (5): 520-30.

10. Coddou E, López J, Madariaga J, Barra M. Sarcoma indiferenciado (embrionario) de hígado en el adulto. Rev Med Chile 2010; 62 (6): 618-22. 